\title{
Healing Rate of Ruminal Incision in Sheep After Single Dose Effect of Low Level Laser Therapy
}

\author{
Qayes Taref Ali ${ }^{1}$, Ahmed Kadhim Munahi ${ }^{1,}$, Mohammed Mahdi Yaseen ${ }^{1}$, Raed Gahat Mehjal ${ }^{1}$, \\ Hassan Al-Karagoly ${ }^{2}$ \\ ${ }^{1}$ Department of Veterinary Surgery, College of Veterinary Medicine, University of Al-Qadisiyah, Diwaniyah, Iraq \\ ${ }^{2}$ Department of Veterinary Pathology, College of Veterinary Medicine, University of Al-Qadisiyah, Diwaniyah, Iraq
}

\section{Email address:}

Qayes.taref@qu.edu.iq (Q. T. Ali), Ahmed.munahi@qu.edu.iq (A. K. Munahi), Mohammed.yaseen@qu.edu.iq (M. M. Yaseen),

Raed.mehjal@gmail.com (R. G. Mehjal), hassan.aliwee@qu.edu.iq (H. Al-Karagoly)

${ }^{*}$ Corresponding author

\section{To cite this article:}

Qayes Taref Ali, Ahmed Kadhim Munahi, Mohammed Mahdi Yaseen, Raed Gahat Mehjal, Hassan Al-Karagoly. Healing Rate of Ruminal Incision in Sheep After Single Dose Effect of Low Level Laser Therapy. European Journal of Clinical and Biomedical Sciences.

Vol. 5, No. 1, 2019, pp. 21-26. doi: 10.11648/j.ejcbs.20190501.15

Received: November 21, 2018; Accepted: December 22, 2018; Published: May 11, 2019

\begin{abstract}
To assess the effect of single dose of low level laser therapy on ruminal healing after rumenotomy, twelve male Awassi local sheep weighing $(30 \pm 5) \mathrm{Kg}$ and aging $(10 \pm 2)$ months, the animals were divided randomly into two groups, (treatment and control groups) each group include six rams equally, collection of venous blood for total white blood cells counts (WBCs) before surgery, 24 hours after surgery, one week and two weeks post operation, all animal subjected to a routine procedure of rumenotomy and after suturing the rumen; the treatment group exposed to a single dose of low-level laser therapy has $820 \mathrm{~nm}, 1000 \mathrm{mw}, 10 \mathrm{sec}, 16 \mathrm{~J} / \mathrm{cmsq}, 146 \mathrm{~Hz}$ pulsing rate per second while the control group had to suture only. The total count of WBCs showed there were elevations in the treatment group when compared with control group in $24 \mathrm{hrs}$ after surgery, a week post operation and two weeks post operation. The histopathological examinations revealed that the healing processes in the treatment group were faster and better exhibited the granulation tissue characterized by vertical formation of blood vessels with the site of incision and illuminated that the newly blood vessels contained RBCs and proliferation of fibroblasts enriched with newly formed blood vessels when compared with the control group which appeared with low in rate and type of healing process after ten days post-surgery. We conclude that Single dose of LLLT is considered as a fast, cheap, and effective technique which could increase healing processes and/or defense mechanisms after rumenotomy in sheep.
\end{abstract}

Keywords: Low-Level Laser, Ruminal Incision, Healing, Sheep

\section{Introduction}

The rumenotomy operation is done in both large and small ruminant animals for treatment of the presence of metallic and non-metallic foreign body [1]. This operation can be done by fixation of rumen by several methods such as skin suture fixation, stay suture technique, Gotza and clamp fixation [2]. Rumen can be fixed by suturing its mucous membrane to decrease spillage of content and infection, Wingarth's ring by which the rumen was pulled out towards the incision and fixation in the dorsal commissure of the skin incision and the other two ends of the pulled part was fixed with the ruminal forceps [3]. The site of operation is determined by the left paralumbar fossa as a perfect site for doing this operation because the muscles of the abdomen shape are thin, so the surgeon should be careful in the opening of this site [4].

The approach of flank laparotomy is the most widely used among small ruminants surgeons for retrieving abdominal and pelvic organs. However, the approach is associated with some challenges such as animals tends to rub the surgical site during healing against available solid objects leading to loosening of sutures and consequently formation of wound dehiscence, prolonged lateral recumbency in ruminants under anesthesia is associated with rumen stasis thereby predisposing the animal 
to bloat and toxemic lactic acidosis [5].

Sheep in many popular arias in Al-Diwaniya province are left free in the streets although it is considered as selective feeder animals and may due to pica and/or mineral deficiencies these animals tend to feed on garbage and other harmful materials such as nylon sacks. The accumulation of such foreign indigestible materials cause intermixing of these substances in the rumen and due to agitating of the rumen leads to precipitation of some of the ruminal contents and salts to form a hard mass occupying a large space in the rumen and consequently impairs digestion. Increases intraruminal pressure especially in pregnant ones and cause emaciation, poor condition and reduction in milk production [6].

Nowadays, most veterinary surgeries are procedures in which the risk to the patients can be precisely evaluated, nevertheless any surgery is a very serious undertaking and even with every care, there are unpredictable hazards or complications [7].

Simple surgical wounds heal through a series of morphological changes, the first step consists in the accumulation of a serious exudates as a results of plasma leakage, exudates are coagulated into a fibrin net which considers as a template for granulation tissue, the latter forms through multiple mechanisms as enhancement of fibroblast proliferation, collagen, and elastin synthesis which form extracellular network of connective tissue, chemotactic factors and beta interferon which newly synthesized by fibroblasts, the abundant extracellular matrix provides more plasticity to the new tissue and permits nutrient diffusion and the whole area is infiltered with macrophages and leucocytes that speed the process of connective tissue regeneration [8].

Primary healing is a process continuing 8-12 days, the sutures can be removed at the completion of this process, while the wound is not yet fully healed, the complete healing lasts three weeks, to reduce the period of healing of the surgical wound and suppress some secondary effects, many therapeutic approaches and drugs are used in postoperative process, one of these methods is Low-Level Laser Therapy (LLLT), the latter has been used as a treatment of wound for more than two decades in the many medical centers of the world $[9,10]$. However, although such wide clinical usage ; there is still controversy regarding the efficacy of LLLT in wound treatment, many laser system (He-Ne, AlGaAs, InGaAlP, etc) [11], different laser parameters (power, wavelength, energy, pulse frequency, pulse duration, etc.) [12], and irradiation conditions (exposure time, frequency and duration of treatment) [13] and due to the great variety of treatment protocols lead to these conclusions [14].

The goal of this research is to present the evaluation of single dose of LLLT role in the surgical aseptic wound of the rumen of sheep based on the hematological and histological examination using laser system (Omega Laser System Ltd. the United Kingdom).

\section{Materials and Methods}

Twelve male Awassi local sheep weighing $(30 \pm 5) \mathrm{Kg}$ and aging $(10 \pm 2)$ months, the animals were fed identical rations and kept under same conditions, before starting the study a physical examination was carried out.

The animals were vaccinated and oral administrated anthelmintic against diseases and divided randomly to 2 groups, (treatment and control groups) each group include 6 rams equally, all animals were deprived of food for 24 hours and water for 12 hours before the operations, all animals restrained in a stock. Collection of venous blood for total white blood cells counts (WBC) before surgery, 24 hours after surgery, one week and two weeks post operation.

The left flank was clipped, shaved and antiseptic applicated, local anesthesia (Lidocaine $\mathrm{HCl} 2 \%$ ) was used in a paravertebral nerve block, the nerves of thoracic (T) 13, lumbar (L) 1,2, and 3 were blocked and the skin was prepared aseptically by using povidone iodine and alcohol solution $70 \%$ alternatively, all the animal were draped, sterile gown and gloves were used. After making laparotomy, the rumen was pulled gently toward the incision suture to the skin using No.1 silk and continuous Connell suture pattern. The suture technique began midway down the caudal side of the incision continued ventral to the ventral commissure to the incision, on the cranial side of the incision dorsally to the dorsal commissure, and finally went to the starting point ventrally (Figure 1).

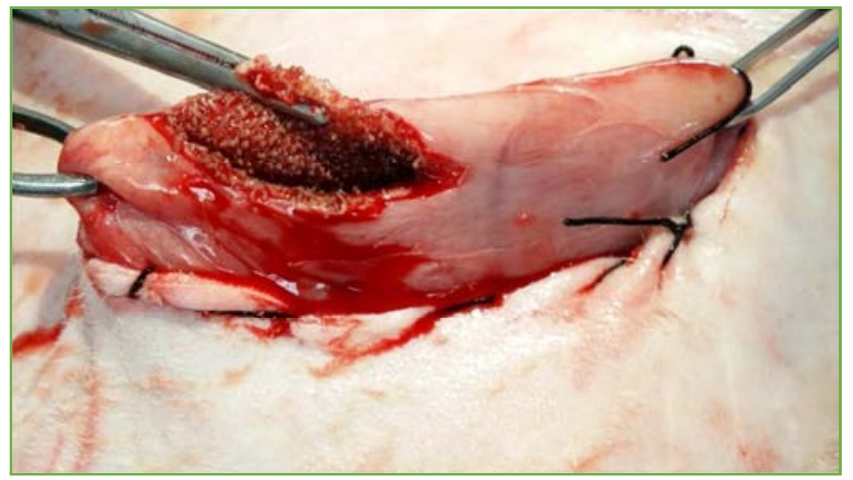

Figure 1. Fixation of rumen with skin by continuous Connell pattern with silk suture.

The suturing material was pulled tightly to invert the skin edges under the rumen for a good sealing. The rumen was incised and explored, then rumenotomy was done with $5 \mathrm{~cm}$ length incision (Figure 2).

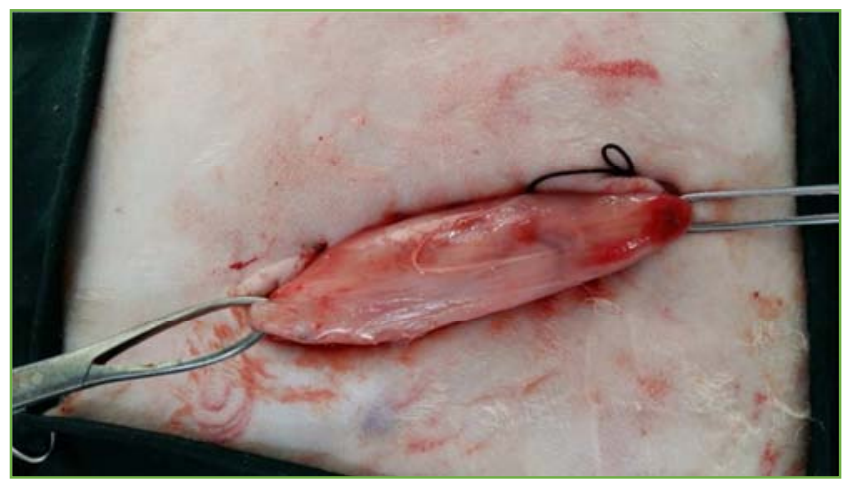

Figure 2. Five centimeters incision length of rumen. 
The incision site was cleaned with sterile normal saline, after that the surgeon scrubbed and regloved, the suturing between rumen and skin was removed, following a final rinse of the rumen with the sterile normal saline. The rumens of the two groups were sutured with Schmedian and Lembert techniques for first and second rows respectively, treatment group subjected to a single dose of low-level laser therapy
(Figure 3) with the following criteria:

$820 \mathrm{~nm}, 1000 \mathrm{mw}, 10 \mathrm{sec}, 16 \mathrm{~J} / \mathrm{cmsq}, 146 \mathrm{~Hz} /$ pulsing rate per second. While the control group closes the rumenotomy routinely. Transverse abdominis muscle and peritoneum were sutured in a simple continuous pattern together by using No.1 chromic catgut.

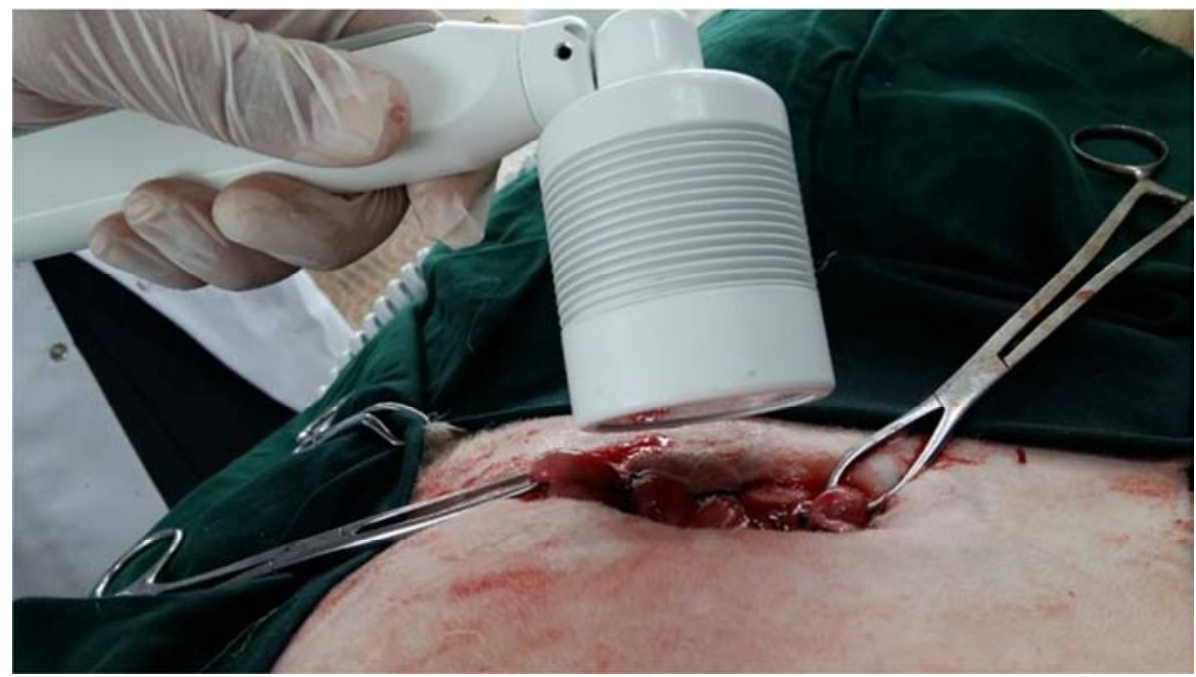

Figure 3. Treatment group subjected to a single dose of low level laser therapy.

Before ending the last suture, the air was forced out of the abdomen by pushing into the opposite flank. The remaining muscular layers (external abdominal oblique and internal abdominal oblique) and subcutaneous fascia were suturing together, the skin was sutured using a horizontal mattress pattern with No.1 silk (Figure 4) $[15,16]$. All animals did not receive medications or antibiotics after surgical operations at all.

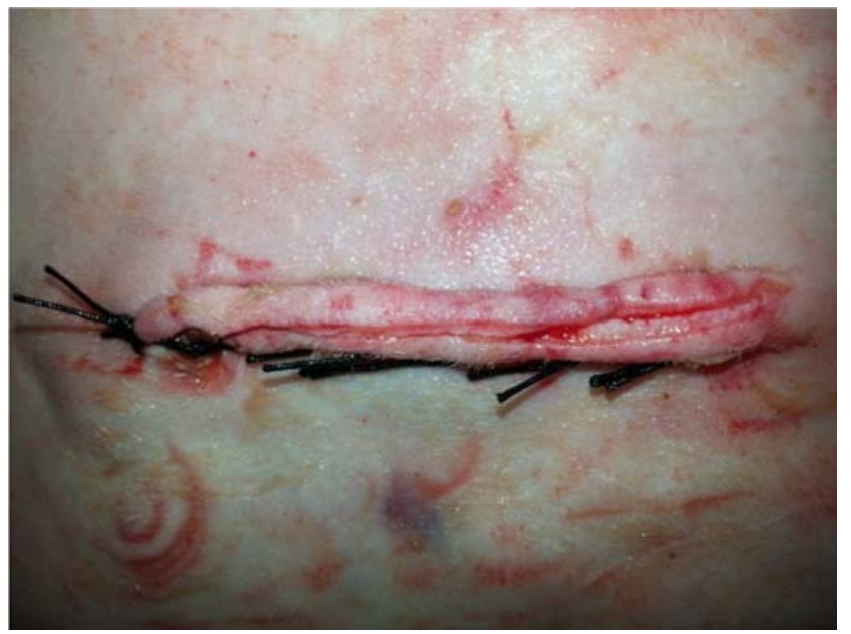

Figure 4. The skin was sutured using a horizontal mattress pattern with No.1 sisilk.
Ten days post operation, the site of the incised rumen of all animals was exposed and biopsies were taken as small as $(1 \mathrm{~cm} 2)$ after that suturing was done for rumen and all layers to close the abdominal cavity, the samples were taken to the histopathological laboratory to evaluate the stages of healing. LSD test was used to compare the significant variances among means $(\mathrm{P}<0.05)$ [17].

\section{Results}

\subsection{Hematological Profile}

There were no variations of total WBCs count of the two groups before operation, as the treatment and control groups recorded $7.13 \pm 0.43$ and $7.24 \pm 0.56$ respectively with no significant difference between them, at 24 hours and at the first week after surgery the both groups having the higher values at all the intervals as well as the control group registered a significant difference $(p<0.05)$ when compared with the treatment group whereas they documented $11.31 \pm$ $0.89,14.21 \pm 0.65$ and $9.65 \pm 0.59,11.23 \pm 0.34$ respectively. The values of the treatment and control groups after two weeks post operation varied slightly from the values before surgery and they hadn't significant differences between them and listed $7.35 \pm 0.55$ and $7.89 \pm 0.11$ respectively, as clarified in table 1 .

Table 1. Total Leucocytes count $\left(\times 10^{3} / \mu \mathrm{ml}\right)$ before and after rumenotomy (mean $\left.\pm \mathrm{SE}\right)$.

\begin{tabular}{llllll}
\hline parameter & group & Before surgery & 24 hrs after surgery & A week after surgery & Two weeks after surgery \\
\hline \multirow{2}{*}{ Total WBCs Count } & Treatment & $7.13 \pm 0.43^{\mathrm{a}}$ & $11.31 \pm 0.89^{\mathrm{a}}$ & $14.21 \pm 0.65^{\mathrm{a}}$ \\
& Control & $7.24 \pm 0.56^{\mathrm{a}}$ & $9.65 \pm 0.59^{\mathrm{b}}$ & $11.23 \pm 0.34^{\mathrm{b}}$ & $7.35 \pm 0.55^{\mathrm{a}}$ \\
\hline
\end{tabular}

Small different letters refer to presence of significance at $\mathrm{p}<0.05$. 


\subsection{Histopathological Evaluation}

After ten days post-surgery; the histopathological changes revealed that the treatment group showed there was a complete healing characterized by marked scar tissue with high proliferation of fibrous connective tissue, also there were formation of new blood vessels (Figure 5).

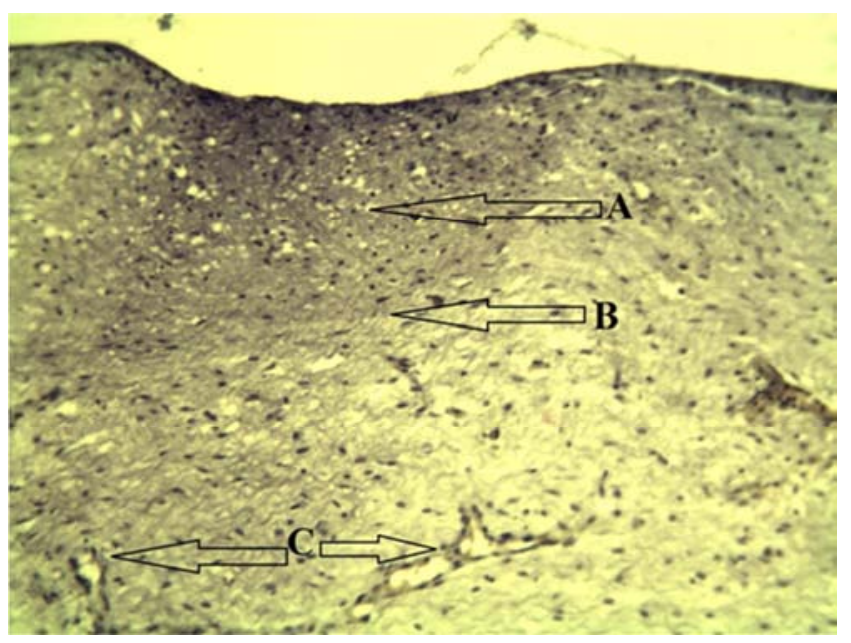

Figure 5. Treatment group after ten days post operation showed there is a complete healing characterized by marked scar tissue (arrow A) with high proliferation of fibrous connective tissue (arrow B), also there is a formation of new blood vessels (arrow C). (50 X H\&E).

As well as the treatment group exhibited the granulation tissue characterized by vertical formation of blood vessels with the site of incision (Figure 6).

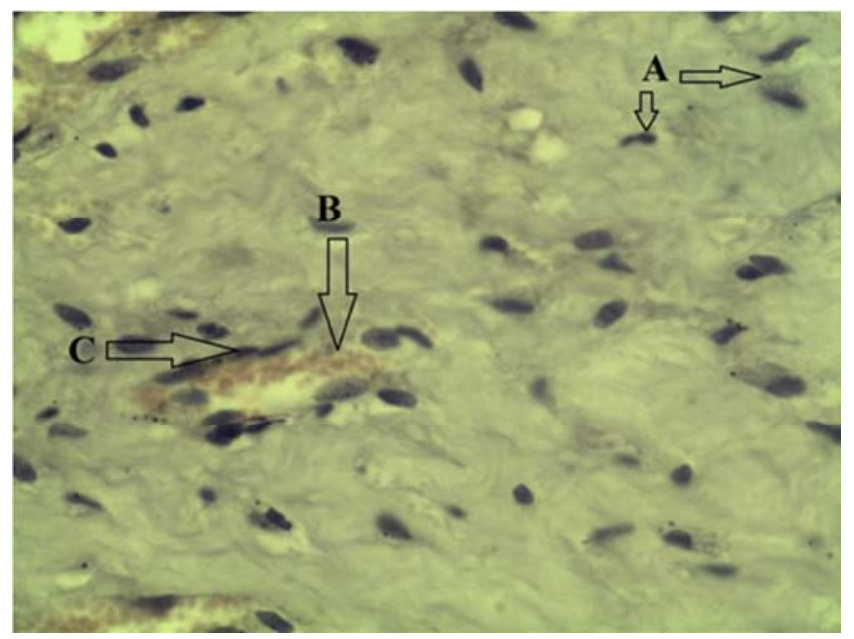

Figure 6. Treatment group after ten days post operation displayed there is a granulation tissue (arrow A) characterized by formation of new blood vessels (arrow B) vertically with the site of incision. (50 X H\&E).

Furthermore, the site of incision in the treatment group illuminated that the newly blood vessels contained RBCs and proliferation of fibroblasts (Figure 7).

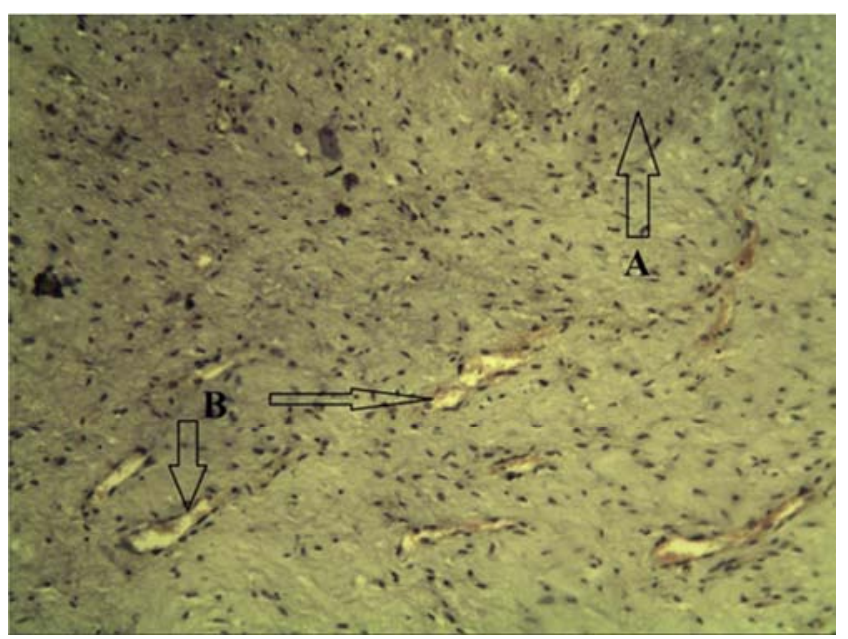

Figure 7. Treatment group after ten days post operation revealed there is a proliferation of fibroblasts (arrow A). There are RBCs (arrow B), within a formation of new blood vessels due to arrangement of endothelial cells (arrow C). (200X H\&E).

The previous description of the treatment group confirmed that the healing processes are well developed in comparison with the control group which demonstrated that there were marked scar tissue as well as profuse fibrosis and few formation of new blood vessels, also there is scattered inflammatory cells (Figure 8).

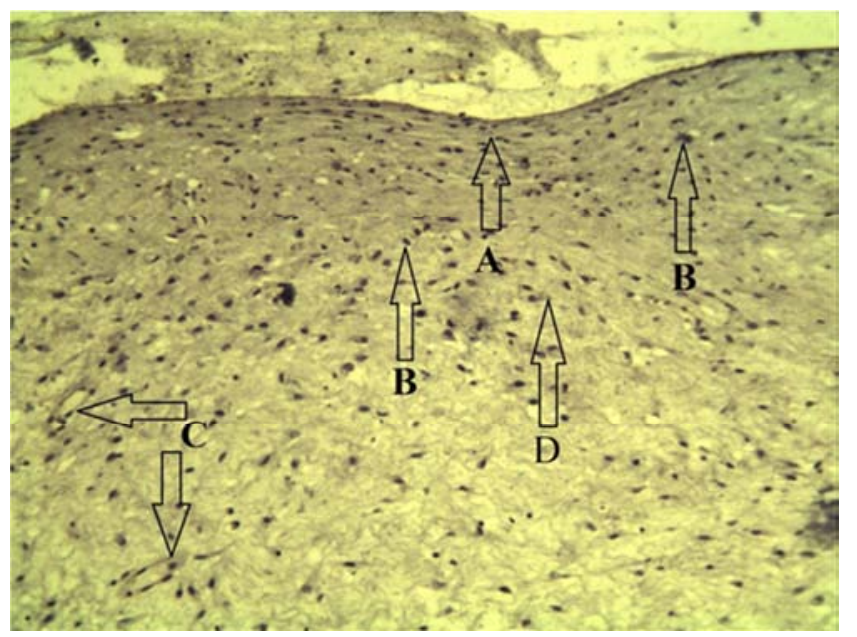

Figure 8. Control group after ten days post operation discovered there is a marked scar tissue (arrow A), there are scattered inflammatory cells (arrow $B$ ), few formation of new blood vessels (arrow C), also there is a profuse fibrosis (arrow D). (50 X H\&E).

In addition, the higher magnification of the control group verified presence of scar tissue and proliferation of fibroblasts with scattered inflammatory cells which mainly were macrophages (Figure 9). 


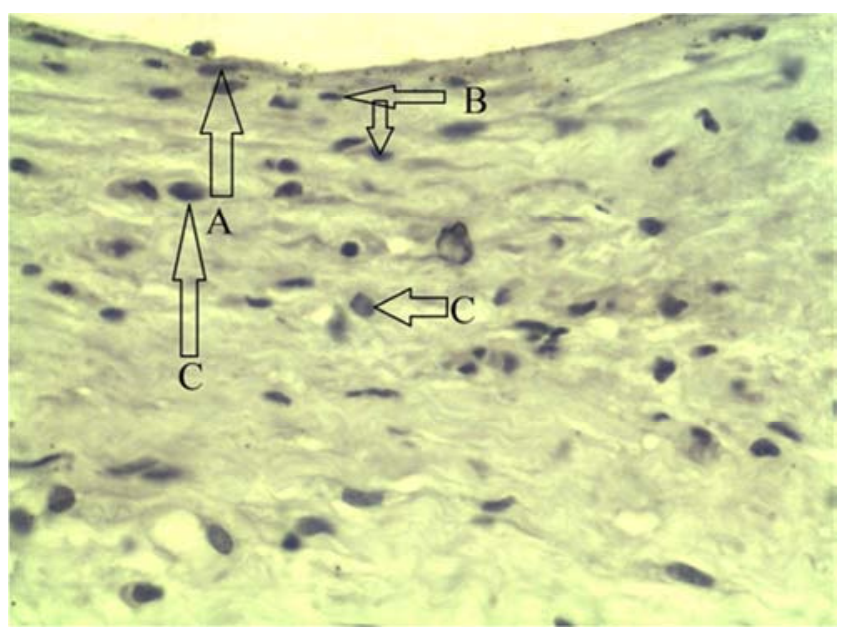

Figure 9. Control group after ten days post operation indicated there is a presence of scar tissue (arrow A), proliferation of fibroblasts (arrow B), and also there are scattered inflammatory cells mainly macrophages (arrow C). (200X $\mathrm{H} \& E)$.

The exploration of the operation site to determine the degree of adhesions between tissues discovered that by using the low level laser therapy lead to less possibility for tissue adhesions, and this technique was quite safe for animals, where the mortality rate was $0 \%$.

\section{Discussion}

Many techniques were used to decrease or diminish contamination and fasten the healing process after rumenotomy, and according to the author's knowledge, information and collecting data, this study is the first one which used single dose of Low Level Laser diode therapy to increase healing process after rumenotomy in sheep.

The hematologic exams have viewed the total count of white blood cells in the experimental and the control groups showed slightly higher values, these dynamics can correlated with the healing process, being a part of the whole regenerative reaction [18]. The high leucocyte levels are generated by the lymphocytes and monocytes both of them were implicated in defense processes locally and generally; monocytes are contributing in phagocytosis in interleukin and lymphocytes are participating in growth factor synthesis [13]. The treatment group exhibited that the total WBCs count increased with moderate elevation when compared with the control group along the period of study and this caused by exposure lo laser therapy and this results accorded with [19]who found that the exposure to low level laser therapy advocates a stimulation of the immune system and the regenerative processes, reflected in monocytosis and in lymphocytosis.

There are several possible explanations for the detected relative elevation of WBCs total count, the topical applied of low power laser irradiation under in vivo conditions may induce systemic effect at least partly because of the expression of some soluble mediators as cytokines from immune competent cells into circulation, a similar mechanism can be in charge of the observed alterations in blood cell counts, another hypothetic explanation for the defined rise of WBCs count could be the induction of alteration in lymphocyte apoptosis rate or decreased expression of intercellular adhesion molecules from endothelial cells avoiding leucocytes from adhesion and subsequent evasion from blood vessels into the tissues, these were adopted by [20] and agreed with the present results of study, who improved that the single laser irradiation can accelerate the recovery of the WBCs count in animals with leucopenia. In contrast, a study was carried out to discover the effect of low level laser therapy on blood flow and oxygen-hemoglobin saturation of skin, [21] established that there was no evidence that the low level laser therapy has a significant instant on the circulation or the oxygen saturation could be found and this differences may returned to the variance in the experimental model and the dose which used in that research.

The biostimulatory effects of the low level laser therapy have been widely reported, and in this study the single dose of the low level laser produced an accelerated healing with better appearance without adherence recorded.

\section{Conclusion}

Irradiation incised and sutured rumen with single dose of $820 \mathrm{~nm}, 1000 \mathrm{mw}, 10 \mathrm{sec}, 16 \mathrm{~J} / \mathrm{cmsq}, 146 \mathrm{~Hz} /$ pulsing rate per second could boosted the recovery of WBCs counts in the peripheral blood and improve the healing processes of the sheep, the combination of multiple exposures might be necessary to progress the immune function, this approach and its mechanism of action need further investigations, we found out that Single dose of LLLT is regarded as a fast, cheap, and effective technique which could increase healing processes and/or defense mechanisms after rumenotomy in sheep.

\section{Acknowledgements}

This research was partially supported by [college of veterinary medicine, university of Al-Qadisiyah]. We thank our colleagues from [department of veterinary surgery] who provided all necessary facilities especially that related with surgical instruments, and [Abbas Ali Al-Laban, assistant professor] for his help in the surgical technique.

\section{Ethical Approval}

All applicable international, national, and/or institutional guidelines for the care and use of animals were followed. All procedures performed in studies involving animals were in accordance with the ethical standards of the institution or practice at which the studies were conducted. We was explane the ethical state of involving animal in our study in details in the materials and method section included the references that we were depended on. 


\section{Disclosure of Potential Conflicts of Interest and Current Submission}

This manuscript has not been previously published and is not under consideration in the same or substantially similar form in any other peer-reviewed media.

\section{References}

[1] Aref, N.-E. M., \& Abdel-Hakiem, M. A. 2013. Clinical and diagnostic methods for evaluation of sharp foreign body syndrome in buffaloes. Veterinary World, 6(9): 586.

[2] Franek, A., Król, P., \& Kucharzewski, M. 2002. Does low output laser stimulation enhance the healing of crural ulceration? Some critical remarks. Medical engineering \& physics, 24(9): 607-615.

[3] Fubini, S., \& Ducharme, N. 2004a. Bovine Surgery In: Farm Animal surgery. Saunders, St. Louise, Missouri._-2004.

[4] Fubini, S., \& Ducharme, N. 2004b. Surgical Management of Umbilical Hernia; Food Animal Surgery, Saunders Publication, an imprint of Elsevier Sciences, Philadelphia, United States of America.

[5] Geehan, A. 2003. Comparative study of Two rumenotomy techniques in caprine. MVSc Thesis College of Veterinary Medicine and Animal Production, Sudan University of Science and Technology.

[6] Haskell, S. 2004. Surgery of the sheep and goat digestive system. Farm animal surgery: 521-526.

[7] Hawkins, D., \& Abrahamse, H. 2006. Effect of multiple exposures of low-level laser therapy on the cellular responses of wounded human skin fibroblasts. Photomedicine and Laser Therapy, 24(6): 705-714.

[8] Hendrickson, D. A. 2013. Techniques in large animal surgery: John Wiley \& Sons.

[9] Heu, F., Forster, C., Namer, B., Dragu, A., \& Lang, W. 2013. Effect of low-level laser therapy on blood flow and oxygenhemoglobin saturation of the foot skin in healthy subjects: a pilot study. Laser therapy, 22(1): 21-30.

[10] Hopkins, J. T., McLoda, T. A., Seegmiller, J. G., \& Baxter, G. D. 2004. Low-level laser therapy facilitates superficial wound healing in humans: a triple-blind, sham-controlled study. Journal of athletic training, 39(3): 223.
[11] Houreld, N., \& Abrahamse, H. 2007. In vitro exposure of wounded diabetic fibroblast cells to a helium-neon laser at 5 and $16 \mathrm{~J} / \mathrm{cm} 2$. Photomedicine and laser surgery, 25(2): 78-84.

[12] Ismail, Z. B., Al-Majali, A., \& Al-Qudah, K. 2007. Clinical and surgical findings and outcome following rumenotomy in adult dairy cattle affected with recurrent rumen tympany associated with non-metallic foreign bodies. American Journal of Animal and Veterinary Sciences, 2(3): 66-71.

[13] Lucas, C., Criens-Poublon, L. J., Cockrell, C. T., \& de Haan, R. J. 2002. Wound healing in cell studies and animal model experiments by Low Level Laser Therapy; were clinical studies justified? A systematic review. Lasers in medical science, 17(2): 110-134.

[14] Mendez, T. M., Pinheiro, A. L., Pacheco, M. T., Nascimento, P. M., \& Ramalho, L. M. 2004. Dose and wavelength of laser light have influence on the repair of cutaneous wounds. Journal of clinical laser medicine \& surgery, 22(1): 19-25.

[15] Petrie, A., \& Watson, P. 2013. Statistics for veterinary and animal science: John Wiley \& Sons.

[16] Qadri, T., Miranda, L., Tuner, J., \& Gustafsson, A. 2005. The short - term effects of low - level lasers as adjunct therapy in the treatment of periodontal inflammation. Journal of clinical periodontology, 32(7): 714-719.

[17] Rocha Júnior, A. M., Vieira, B. J., Andrade, L. C. F. d., \& Aarestrup, F. M. 2007. Effects of low-level laser therapy on the progress of wound healing in humans: the contribution of in vitro and in vivo experimental studies. Jornal Vascular Brasileiro, 6(3): 257-265.

[18] Schwartz, A. J., Wilson, D. A., Keegan, K. G., Ganjam, V. K., Sun, Y., Weber, K. T., \& Zhang, J. 2002. Factors regulating collagen synthesis and degradation during second-intention healing of wounds in the thoracic region and the distal aspect of the forelimb of horses. American journal of veterinary research, 63(11): 1564-1570.

[19] Scott, R. 2005. Caprin surgery and application. Manoa USA.

[20] Weaver, A. D., Atkinson, O., Jean, G. S., \& Steiner, A. 2018. Bovine surgery and lameness: John Wiley $\&$ Sons.

[21] Zhao, J., Cheng, K., Deng, H., Zhao, L., Liu, L., Guo, M., Zhang, H., \& Shen, X. 2014. The effect of different laser irradiation on cyclophosphamide-induced leucopenia in rats. Evidence-Based Complementary and Alternative Medicine, 2014. 